\title{
Predictive DNA-testing for Huntington's disease and reproductive decision making: a European collaborative study
}

\author{
Gerry Evers-Kiebooms*,1, Kurt Nys ${ }^{1}$, Peter Harper $^{2}$, Moniek Zoeteweij $^{3}$, Alexandra Dürr ${ }^{4}$, \\ Gioia Jacopini ${ }^{5}$, Christos Yapijakis ${ }^{6}$ and Sheila Simpson ${ }^{7}$
}

${ }^{1}$ Center for Human Genetics, Leuven, Belgium; ${ }^{2}$ Institute of Medical Genetics, Cardiff, UK; ${ }^{3}$ Department of Clinical Genetics, Leiden, The Netherlands; ${ }^{4}$ Département de génétique, cytogénétique et embryologie, Paris, France; ${ }^{5}$ Istituto di Psicologia, Rome, Italy; ${ }^{6}$ Department of Neurology, Athens, Greece; ${ }^{7}$ Clinical Genetics, Aberdeen, UK

This European collaborative study addresses the question whether a predictive test result for Huntington's disease (HD) has an effect on subsequent reproduction by comparing carriers and non-carriers of the Huntington mutation. A unique characteristic of this study is that this evaluation is done in persons at reproductive age who had a predictive test after the identification of the Huntington gene and who were counselled in one of the participating centres. Data were collected for 180 carriers and 271 non-carriers who received a predictive test result in the period 1993-1998 in Aberdeen, Athens, Cardiff, Leiden, Leuven, Paris or Rome. The mean age of the total study group was $\mathbf{3 1 . 5}$ years and for about half of the group the follow-up interval was 3 years or more, with a maximum of 7 years. The collaborative study clearly revealed an overall impact of the predictive test result on subsequent reproduction: $14 \%$ of the carriers had one or more subsequent pregnancies vs $\mathbf{2 8} \%$ of the non-carriers. In the total carrier group a prenatal test was carried out in about two thirds of the pregnancies and one child was born after preimplantation genetic diagnosis; artificial insemination by donor, egg cell donation or adoption were not reported. A more refined analysis was performed in the subgroup with a follow-up interval of at least 3 years and who reported 'family planning' as a motive to apply for predictive testing in the pretest period. The complexity of this motive is discussed. In this subgroup with a desire for children in the pretest period the effect of the predictive test result was more pronounced: $69 \%$ of the non-carriers had subsequent pregnancies while only $39 \%$ of the carriers who mentioned 'family planning' as one of the major reasons to apply for predictive testing had a subsequent pregnancy. Of the carriers with one or more subsequent pregnancies the percentage using prenatal diagnosis was slightly higher than the percentage not using it, although there were clear differences from one centre to another. The latter group's decisions may seem more intriguing but may be partially understood based on stage theories of health behaviour. Last, but not least, whatever option is chosen by a couple at increased risk of transmitting the Huntington mutation, it is of the utmost importance that professionals fully respect this decision and support the couple.

European Journal of Human Genetics (2002) 10, $167-176$. DOI: 10.1038/sj/ejhg/5200781

\footnotetext{
*Correspondence: Prof. Gerry Evers-Kiebooms, Psychosocial Genetics Unit, Center for Human Genetics K.U.Leuven, Herestraat 49, 3000 Leuven, Belgium. Tel: 32-16-345867; Fax: 32-16-346044;

E-mail: Gerry.Kiebooms@med.kuleuven.ac.be

Received 12 October 2001; revised 21 December 2001; accepted 4 January 2002
} 
Keywords: Huntington's disease; predictive testing; prenatal testing; preimplantation genetic diagnosis; family planning; reproductive decision making

\section{Introduction}

Huntington's disease (HD) is currently an untreatable progressive neuropsychiatric disorder, characterised by involuntary movements, neuropsychological defects and personality changes. The mean age at onset is about 40 years. Symptoms progress slowly with death occurring an average of 15 years after the disease onset. Huntington's disease is inherited as an autosomal dominant trait, with the gene localised on the short arm of chromosome 4. In March 1993 the Huntington-gene was isolated containing an expanded and unstable trinucleotide repeat (CAG) in HD patients. ${ }^{1}$ The localisation and subsequent identification of the gene resulted in the offer of a predictive DNA-test to asymptomatic persons belonging to families with Huntington's disease. In a recent study of Harper, Lim and Craufurd, ${ }^{2}$ on behalf of the UK Huntington's Disease Prediction Consortium, 'the normal range was classified as up to 30 repeats, with 39 or more repeats as abnormal, the small intervening number being considered as 'equivocal' (p. 568). Patients with a predictive test result in the latter category are also called patients with an intermediate allele; this result is quite exceptional $( \pm 1.5 \%$ of the persons with a predictive test in the UK in the period 1988-1997).

The availability of a predictive test for HD in the second half of the '80s was an important milestone in medical history as well as a challenge for families and professionals. It confronted members from families with HD with the choice 'to know' or 'not to know', a decision with important short-, mid and long-term consequences. Because of the particular nature of predictive tests for (currently) untreatable late onset diseases, a large amount of international debate and consultation preceded the implementation of the first predictive DNAtests in clinical practice. Guidelines were elaborated by an ad hoc committee of the International Huntington Association and the World Federation of Neurology. ${ }^{3}$ Predictive test requests are often approached by a multidisciplinary team consisting of a clinical geneticist, a neurologist, a psychologist and/or a social worker and/or a genetic nurse. During the pretest counselling sessions, full information is provided on $\mathrm{HD}$ and on the predictive test. The role and psychological meaning of the disease and the test in the course of life of the testee are explored. It is the main aim of the pretest counselling sessions to help people to use sufficient time for reflection, to develop a scenario of their life after a favourable test result, after an unfavourable test result or without having a predictive test and to make a free informed decision about having or not having a predictive test. After the disclosure of the predictive test result, short- and long-term emotional and social support is systematically provided during follow-up counselling sessions.
All over the world the proportion of tested people is smaller than could be expected based on intentions and attitudes before the availability of the test. ${ }^{4-10}$ The estimated uptake seems to be lower than $20 \%$ in most European countries, in the US, in Canada and Australia. It is very hard to unravel why only a minority of individuals at risk have chosen to be tested, while most others prefer not to be tested. ${ }^{7}$ It is obvious that demographic variables hardly play any part in the decision. Decruyenaere et al. ${ }^{11}$ assessed the perceived benefits or reasons for taking the test and the perceived barriers against taking the test in tested and untested persons belonging to the same sibships. The most important benefits or reasons for taking the test are: the need for certainty or relief from uncertainty, making reproductive decisions, informing their children about their risk for $\mathrm{HD}$ and making decisions on practical matters (financial, employment). Important barriers against the test are: the anticipated inability to cope with a bad test result, the feeling that important decisions do not have to depend on a test result, being happier when not knowing than with the certainty of a bad result, the lack of a treatment, concern about the reaction of their children and partner, and finally the pretest counselling sessions. The reported benefits and barriers are confirmed in other studies. ${ }^{5,12}$ In some countries, concern about life insurance is an additional barrier to take the test. ${ }^{13}$

The motivation to apply for predictive testing is very complex: there is often more than one reason and there are conscious as well as unconscious motives. It is important to keep in mind that in most studies only the test applicants' conscious motives expressed during counselling sessions or the reasons indicated in a questionnaire by persons at risk for $\mathrm{HD}$ are used for analysis. The major motives for requesting predictive testing are 'certainty for the own future', 'family planning' and 'informing the children already born/have information about their risk'. The perceived role of the latter two reasons is of course strongly dependent on the age and family situation of the test applicants. Many authors have drawn attention to the fact that test applicants who mention 'family planning' in the pretest period as one of the major reasons for having a predictive test and who receive an unfavourable test result are confronted with new decision difficulties and additional emotional burden. ${ }^{7,14-18}$ In principle carriers of the HD mutation with a strong desire to have children have the following options: refraining from having children, taking the risk of having a child with the Huntington mutation, using prenatal diagnosis, artificial insemination with donor sperm, IVF with donor eggs, preimplantation genetic diagnosis (PGD) and adoption. The use of PGD for HD is discussed by Geraedts and Liebaers. ${ }^{19}$ 
In contrast to the large number of studies about the impact of predictive testing on psychological wellbeing ( $c f$. EversKiebooms et $a l^{20}$ for a review on the impact of predictive testing on the psychological wellbeing of testees and their family), systematic studies on reproductive choices in HD families after the introduction of predictive and prenatal DNA-testing are scarce. There are almost no systematic studies on reproductive choices after a predictive test result, notwithstanding the fact that predictive testing for HD and prenatal testing for HD as two separate issues have received a lot of attention. Moreover the few available publications have small samples and/or a short follow-up interval or consist of case presentations. In Manchester, UK, $81 \%$ of the 109 'potential users' said they would request prenatal diagnosis if pregnant, while only three couples had a prenatal test. ${ }^{4}$ In Wales, UK, $17 \%$ of 90 couples referred to the genetic centre for information about exclusion testing in the period 1986-1989, had one or more prenatal exclusion tests. ${ }^{14}$ After a positive test result all pregnancies have been terminated. The most important reason why prenatal diagnosis was not accepted by the other couples was objection to pregnancy termination for HD. Adam et al ${ }^{15}$ reported on 'intentions to use' prenatal diagnosis and 'use' of prenatal testing in the group of subjects who had entered the Canadian Collaborative Study on predictive testing before September 1991. Overall $43 \%$ had the intention to use prenatal testing if they or their spouse were pregnant. In the period of the study $18 \%$ of the 38 couples who did not receive a decreased risk and who had one or more pregnancies in the study period used prenatal diagnosis. All but one increased risk pregnancies were terminated. The most frequent reason given for declining prenatal testing was the hope that a cure would be found in time (about $80 \%$ of the candidates). Other reasons were: reluctance to pregnancy termination, concern about the safety of the prenatal procedure and wanting to determine own status before deciding about prenatal testing. Subjects who already had children and practicing members of a religious organisation were less likely to choose prenatal diagnosis.

The present study reports on a systematic European collaborative study about reproductive decision making after predictive testing for $\mathrm{HD}$, based on the results of seven centres in six European countries. The first and main objective of the study is to compare post predictive test reproductive decision making and reproduction of carriers and non-carriers of the Huntington mutation who were at reproductive age when they received the predictive test result and to evaluate the uptake of prenatal testing in the former group. Secondly the study is aimed at analysing the differences in reproductive history after predictive testing between the participating centres. The other part of the European Collaborative Study carried out in the context of a European Commission funded project (BIOMED-project No ERB BMH4 CT98-3926), concerns two other types of data: all prenatal tests (full prenatal testing and exclusion testing) performed in the same seven centres since the start of their programme and all prenatal tests performed in their country since the identification of the Huntington gene in 1993.

\section{Methods \\ Design of the study}

Seven genetic centres from six different European countries collected data about the reproductive history of persons who had a predictive test for HD in their centre: Leuven, Leiden, Cardiff, Aberdeen, Paris, Rome, and Athens. Most of these centres have used a multidisciplinary approach ( $c f$. supra) since the start of their predictive test programme. To be eligible for the European Collaborative study testees had to meet the following criteria: (1) the testee had to be 45 years or younger at the time of the communication of the test result; (2) the testee had to be tested and counselled in one of the seven centres, and (3) the communication of the predictive test result had to be done after 1992 and before 1999 .

For each testee meeting the inclusion criteria of the study the following information was collected by means of a three sheet questionnaire developed for this purpose: (1) date and result of the predictive test; (2) socio-demographic data; (3) reasons for predictive testing based on the files from the pretest period; (4) information about the last contact with the testee; (5) pregnancies; (6) adoptions; (7) presence of a pregnancy at the time of communicating the test result; (8) perception of completeness of the family; and (9) intentions for PGD. For most topics multiple choice questions were used. Information was usually retrieved from the files of the genetic centres containing data from the pretest sessions as well as the post-test follow-up sessions. When feasible the testee was contacted by the genetic centre for updating the data, thereby always respecting testees' choice to have no further follow-up contacts (the latter was only the case for a small minority). In the genetic centre in Leiden, The Netherlands, a slightly different approach was used. In contrast to the other centres participating in the European study, not all testees meeting the eligibility criteria were included in the study, but only a subgroup of them, namely those testees who participated in another ongoing study in Leiden, involving neuropsychological testing. There are no reasons to assume that this subgroup would be biased in a way that interferes with the collaborative study. The main disadvantage of this different approach in Leiden is the fact that data could only be collected for part of the eligible group of testees.

All the questionnaires filled in by professionals of the seven centres were sent to the coordinating center in Leuven, Belgium, where the data were centralised and analysed. Respect for the privacy of the testees was ensured by anonymising all the data before sending them to the coordinating centre. There was only an identification number on the forms sent to Leuven. Two annual workshops involving all the partners of the European project led to a critical discussion of preliminary analyses. 
The SAS software package was used for the description and analysis of all data. ${ }^{21}$ Data analysis mainly consisted of descriptive statistics. Chi-squares were used to test associations between variables measured at nominal level (discrete categories). Nothwithstanding the dichotomous nature of the dependent variable (having or not having pregnancies after the communication of the predictive test result), the authors preferred the technique of analysis of covariance above logistic regression analysis to test the hypotheses they wanted to test in this empiric study. A first analysis of covariance was performed to test the effect of the independent variable 'predictive test result' (carrier $v s$ non-carrier of the Huntington mutation) on the dependent variable 'having or not having subsequent pregnancies', whereby the continuous variables 'age of the prospective parent belonging to the family with $\mathrm{HD}^{\prime}$ and 'number of children before applying for predictive testing' were used as covariates. A second analysis of covariance was carried out to test the main effect of two independent variables - 'predictive test result' and 'sex of the prospective parent belonging to the family with HD' - as well as their interaction effect on the dependent variable 'having or not having subsequent pregnancies', hereby using the same two covariates as in the previous analysis.

\section{Description of the study group}

Testees' carrier status, age and motives to apply for predictive testing The total study group consists of 451 testees: 180 carriers, 271 non-carriers (two testees with an intermediate allele were not included in the study). Table 1 gives an overview of the results of the predictive tests per centre. All the participating centres had more predictive tests with a 'no carrier' result than with a 'carrier' result. In the total group $60 \%$ of the testees was a non-carrier and $40 \%$ was an asymptomatic gene carrier.

The distribution of the year of the communication of the predictive test result is compatible with the general trend of increased uptake in the first year after the identification of the gene: 79 in 1993, 97 in 1994, 76 in 1995, 78 in 1996, 54 in 1997 and 67 in 1998. The lower numbers for the last years are

Table 1 Composition of the study group: centre and carrier vs non-carrier of the Huntington mutation

\begin{tabular}{lcc}
\hline Centre & Carrier & Non-carrier \\
\hline Leuven (Belgium) & 28 & 39 \\
Leiden (The Netherlands) & 19 & 39 \\
Cardiff (UK) & 23 & 37 \\
Aberdeen (UK) & 29 & 32 \\
Paris (France) & 38 & 46 \\
Rome (Italy) & 10 & 19 \\
Athens (Greece) & 33 & 59 \\
Total group & 180 & 271
\end{tabular}

partially due to the fact that predictive testing steadily became available in an increasing number of genetic centres in five of the six participating countries. There were slightly more female testees ( $58 \%$ ) than male testees ( $42 \%)$. The mean age of the testees meeting the criteria for the study was 31.5 years $(\mathrm{SD}=7$; range $=[18-45])$. The information from each centre is given in Table 2 . The majority of the testees (77\%) had a stable relationship at the time of the communication of the test result. The mean age of the partner of the testee $(n=298)$ was 33.3 years $(\mathrm{SD}=7.7$; range $=[18-61])$. Overall about half of the testees were 31 years of age or younger when they received the predictive test result.

The most frequently mentioned reason to apply for predictive testing (expressed in the pretest period) was 'having certainty' or 'getting rid of uncertainty': $81 \%$ of the testees expressed this reason as one of the major motives to apply for predictive testing for HD. 'Reproductive decision making' was mentioned by $38 \%$ of the testees as one of the major reasons and 'informing the children' by $28 \%$. Eleven per cent mentioned other motives for predictive testing. The sum of the percentages exceeds $100 \%$ because it was possible to indicate more than one major reason. Taking into account the fact that about half of the testees in the study group were 31 years or younger at the time of the communication of the predictive test result, the percentage of testees mentioning family planning as a motive seems rather low.

Pregnancies before the communication of the predictive test result About half of those who were revealed to be carriers and about half of those who were revealed to be noncarriers already had one or more pregnancies before applying for predictive testing (see Table 3). Overall there were 442 pregnancies before the predictive test. One hundred and eighty carriers of the Huntington mutation had 177 pregnancies, 271 non-carriers had 265 pregnancies. These pregnancies resulted in the birth of 388 children, in 32 miscarriages, and 22 terminations. In the total study group nine testees had one or more pregnancies with prenatal diagnosis for HD before the communication of the predictive test result (mainly exclusion tests). For only one couple the use

Table 2 Mean age of the testee at the time of the communication of the predictive test result for each centre

\begin{tabular}{lcc}
\hline Centre & Mean & SD \\
\hline Leuven & 29.6 & 6.9 \\
Leiden (The Netherlands) & 32.3 & 7.3 \\
Cardiff (UK) & 33.0 & 7.4 \\
Aberdeen (UK) & 31.0 & 7.7 \\
Paris (France) & 32.2 & 6.7 \\
Rome (Italy) & 31.3 & 6.1 \\
Athens (Greece) & 29.9 & 6.7 \\
Total group & 31.5 & 7.0 \\
\end{tabular}


Table 3 Number of pregnancies per testee before the communication of the predictive test result

\begin{tabular}{lccc}
\hline $\begin{array}{l}\text { Number of } \\
\text { pregnancies }\end{array}$ & $\begin{array}{c}\text { Carrier group } \\
(\%) \\
(\mathrm{n}=180)\end{array}$ & $\begin{array}{c}\text { Non-carrier } \\
(\%) \\
(\mathrm{n}=271)\end{array}$ & $\begin{array}{c}(\%) \\
(\mathrm{n}=451)\end{array}$ \\
\hline No prior pregnancies & 47 & 51 & 50 \\
One pregnancy & 21 & 16 & 18 \\
Two pregnancies & 22 & 20 & 20 \\
Three pregnancies & 6 & 9 & 8 \\
Four pregnancies & 4 & 4 & 4 \\
& 100 & 100 & 100 \\
\hline
\end{tabular}

of artificial insemination was reported and for one other couple adoptions were reported. In this type of study it is very hard to make a judgement about the completeness of the data on adoption, artificial insemination by donor and egg cell donation. The number of pregnancies before the communication of the result of the predictive test was significantly correlated with the presence of decision making as a reason to apply for predictive testing $(r=-0.31 ; P<0.001)$ : as could be expected the more pregnancies there were before the predictive test, the less frequent reproductive decision making was mentioned as a motive for applying for predictive testing. In the group without any pregnancy before the predictive test the number of testees mentioning reproductive decision making as a reason to apply for predictive testing was comparable to the number of testees not mentioning reproductive decision making in the pretest period. From a methodological point of view it is important to keep in mind that a pregnancy at the time of the communication of the predictive test result was considered as a pregnancy in the pretest period, no matter what the outcome of the pregnancy was (birth, miscarriage or termination). As a consequence all these pregnancies were integrated in the data regarding reproductive history before predictive testing (although the outcome of the pregnancy may only be visible after the communication of the test result). There were 14 couples with a pregnancy at the time of the communication of the predictive test result (3.5\% of the total group of testees). In four of the couples one of the partners revealed to be carrier (29\%), in the other couples the testee revealed to be a noncarrier. In half of the couples the mother belonged to a Huntington family, in the other half the father.

The length of the follow-up interval after the communication of the predictive test result For more than two-thirds of the study group the most recent contact with the testee took place in 1999 (47\%) or $2000(21 \%)$. The follow-up period was calculated as the difference between the date of the communication of the predictive test result and the reported date of the last contact in the returned questionnaire. For almost half of the testees the follow-up period is 3 years or longer with a maximum of 7 years, for $13 \%$ between 2 and 3 years, for $19 \%$ between 1 and 2 years and for $19 \%$ of the testees the follow-up interval was less than 1 year. So the follow-up interval in this study seems adequate (although not optimal) for studying the reproductive history of the testees after predictive testing. However it is important to keep in mind that the families should not be considered as 'complete': it was reported that $43 \%$ of the testees considered their family as complete at the time of the last follow-up contact, whereas $27 \%$ considered it incomplete at that time and $14 \%$ were still undecided about having (additional) pregnancies or not (for $16 \%$ of the testees this information was unknown or unavailable).

\section{Results}

An overall picture of post predictive test reproduction For 103 of the 451 testees one or more pregnancies were reported for the follow-up interval after the communication of the predictive test result (see Table 4). These 142 pregnancies resulted in the birth of 104 children, in 22 miscarriages, and in 16 terminations (including one termination in the non-carrier group for another reason than HD). In the group of carriers 26/180 (14\%) had one or more pregnancies and 15 of them had one or more prenatal tests. As could be expected the percentage of non-carriers with one or more pregnancies is much higher: $77 / 271$ (28\%) had one or more pregnancies. For the 40 pregnancies in the carrier group 24 prenatal tests for HD were performed: nine prenatal tests had a favourable result and 15 an unfavourable. Twelve pregnancies in the carrier group resulted in the birth of a child, without a prenatal test. There were three miscarriages in this group and one preimplantation genetic diagnosis. The use of other alternatives (artificial insemination by donor, egg cell donation or adoption) was not reported.

Reproductive history of carriers and non-carriers who expressed family planning as a motive in the pretest period

When comparing the post predictive test reproductive history of the total group of carriers and the total group of non-carriers it is obvious that the former group is less

Table 4 An overall picture of post predictive test reproduction as a function of carrier status

\begin{tabular}{lcc}
\hline $\begin{array}{l}\text { Number of } \\
\text { pregnancies }\end{array}$ & $\begin{array}{c}\text { Carrier (\%) } \\
(n=180)\end{array}$ & $\begin{array}{c}\text { Non-carrier (\%) } \\
(n=271)\end{array}$ \\
\hline & & \\
No post test pregnancies & 85 & 72 \\
One post test pregnancy & 9 & 22 \\
Two post test pregnancies & 4 & 3 \\
Three post test pregnancies & 1 & 3 \\
Six post test pregnancy & 1 & 0 \\
& 100 & 100 \\
\hline
\end{tabular}


inclined to engage in a pregnancy. However it is very important to look at the data in a more 'refined' way, thereby taking into account 'reproductive decision making' as a motive for predictive testing. Therefore we focus our attention on the subgroup who mentioned reproductive decision making as one of the major motives in the pretest period (see Table 5).

In the subgroup of 57 carriers who mentioned reproductive decision making as a motive for predictive testing for HD in the pretest period 18 carriers (32\%) had pregnancies after the predictive test. The total number of pregnancies of these 18 carriers was 31 . Ten carriers had one pregnancy, six had two, one had three, and one carrier had six post predictive test pregnancies. Eleven carriers had one or more pregnancies with prenatal diagnosis and the other seven carriers had none of their pregnancies tested. For the total of 31 pregnancies in the carrier group 20 prenatal tests were performed leading to eight favourable results and 12 unfavourable results; the latter 12 pregnancies were terminated. In seven pregnancies no prenatal test was performed. Three pregnancies resulted in a miscarriage and one pregnancy occurred after IVF and preimplantation genetic diagnosis.

The following patterns were found in the subgroup of 11 carriers with at least one prenatal diagnosis after predictive testing. Ten carriers or their spouse had a prenatal test in every pregnancy after the communication of the predictive test result: five testees had one pregnancy, four had two and one had three pregnancies tested by means of prenatal testing. In the eleventh testee a more complicated pattern was found. This testee had six pregnancies after the communication of the predictive test result. After an unfavourable prenatal test result in the first pregnancy, a miscarriage in the second pregnancy, three more unfavour-

Table 5 Post predictive test reproduction of carriers and non-carriers of the Huntington mutation who expressed family planning as a motive for applying for predictive testing in the pretest period

\begin{tabular}{|c|c|c|c|}
\hline & $\begin{array}{l}\text { Carriers } \\
(\mathrm{n}=57)\end{array}$ & $\begin{array}{l}\text { Non-carriers } \\
(n=114)\end{array}$ & $\begin{array}{c}\text { Total } \\
(\mathrm{n}=171)\end{array}$ \\
\hline Number of testees with pregnancies & $18(32 \%)^{b}$ & $64(56 \%)^{c}$ & 82 \\
\hline Number of pregnancies & 31 & 88 & 119 \\
\hline Number of births (with PGD) ${ }^{\mathrm{a}}$ & $16(1)$ & 68 & $84(1)$ \\
\hline Number of miscarriages & 3 & 19 & 22 \\
\hline Number of terminations & 12 & 1 & 13 \\
\hline $\begin{array}{l}\text { Number of testees with } \\
\text { prenatal tests }\end{array}$ & $11(19 \%)^{b}$ & & \\
\hline Number of prenatal tests & 20 & & 20 \\
\hline Number of favourable results & 8 & & 8 \\
\hline Number of unfavourable results & 12 & & 12 \\
\hline
\end{tabular}

${ }^{\text {aPGD }}=$ Preimplantation Genetic Diagnosis; ${ }^{b}$ percentage calculated with the total number of carriers $(n=57)$ as denominator; cpercentage with the total number of non-carriers $(n=114)$ as denominator. able prenatal test results in the third, fourth and fifth pregnancy, the testee decided to make use of a preimplantation genetic diagnosis. This sixth pregnancy resulted in the birth of a child without the Huntington mutation.

In the subgroup of 114 non-carriers who mentioned reproductive decision making as a motive for predictive testing in the pretest period $56 \%$ of the testees had one or more pregnancies after the communication of the predictive test result. The total number of pregnancies after the predictive test in this group was 88 . Forty-eight couples had one pregnancy, eight couples had two and eight couples had three further pregnancies.

A comparison of the post predictive test reproduction of carriers and non-carriers with reproductive decision making as a motive reveals a considerable difference: in the former group 18/57 (32\%) engage in at least one pregnancy against $64 / 114(56 \%)$ in the latter group. The difference between carriers and non-carriers is significant $\left(\chi^{2}=9.11 ; P<.01\right)$ The fact that 19 out of the 88 pregnancies (22\%) of the noncarriers with family planning as a motive for predictive testing resulted in a miscarriage (eight testees had one miscarriage, four had two and one testee had three miscarriages) is a finding without a clear explanation.

Notwithstanding the fact that the numbers become rather small an additional more refined analysis was performed on the subgroup of testees with a follow-up interval of at least 3 years and with reproductive decision making as a major motive to apply for predictive testing. This is indeed the most appropriate group to evaluate the effect of the predictive test result on reproduction. This group consists of 96 testees, $40 \%$ carriers and $60 \%$ non-carriers. In the group of carriers $(n=38)$ there were 15 people (39\%) with pregnancies after the predictive test. The total number of pregnancies of these 15 people was 25 . These pregnancies resulted in 14 births, three miscarriages and eight terminations. Eight of the 15 testees had one or more prenatal tests. In this group there were 15 prenatal tests performed, resulting in seven favourable and eight unfavourable results. In the group of non-carriers ( $n=58$ ) 69\% had further pregnancies. The 58 pregnancies in this group resulted in 43 births, 14 miscarriages and one termination (for other reasons than for HD). A comparison of the percentage of testees engaging in one or more pregnancies in the non-carrier group and the carrier group with family planning as a motive in the pretest period reveals that the percentage is much higher in the former group (69\%) than in the latter group (39\%). The difference between carriers and non-carriers is significant $(\chi=8.23 ; P<0.01)$.

Post predictive test reproductive history of carriers and non-carriers with family planning as a motive expressed in the pretest period: data per centre

Table 6 gives a detailed picture of each centre of post predictive test reproductive history of carriers who mentioned reproductive decision making as a motive to apply for predictive testing in the pretest period. We now only consider 
Table 6 Post predictive test reproduction of carriers of the Huntington mutation who expressed family planning as a motive for applying for predictive testing in the pretest period: data per centre ${ }^{\mathrm{a}}$

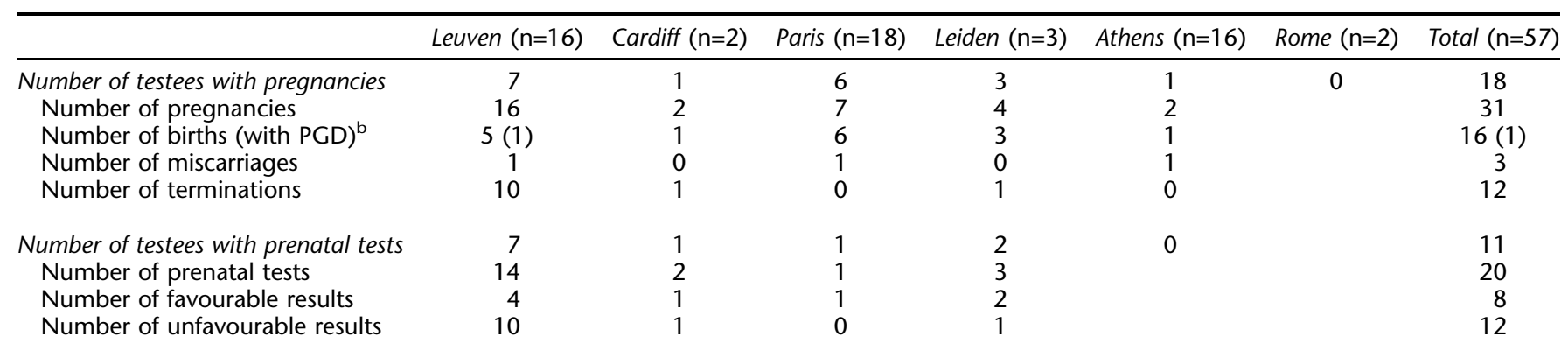

${ }^{\mathrm{a}}$ In Aberdeen three were no carriers who expressed family planning as a motive for applying for predictive testing in the pretest period; ${ }^{\mathrm{b}} \mathrm{PGD}=$ Preimplantation Genetic Diagnosis.

the three centres with more than five testees in this situation. In the genetic centre of Leuven all carriers with family planning as a motive who had further pregnancies $(n=7)$ used prenatal testing. In Paris the proportion of carriers using prenatal testing was clearly lower. In Athens there was only one testee with further pregnancies and no prenatal diagnosis was used.

The same type of data for non-carriers with family planning as a motive in the pretest period is given in Table 7. The proportion of testees engaging in at least one pregnancy in the non-carrier group with family planning as a motive in the pretest period is rather similar in Leuven and Paris, but clearly different in the other centers with a sufficiently large number of testees in this situation. The proportion is clearly highest in Athens.

\section{A definite impact of the predictive test result on reproduction}

In the total group of testees who had a predictive test for HD 'having or not having pregnancies' after the communication of the predictive test result is significantly correlated with the test result: $r=0.16(P<.001)$. The correlation is higher in the subgroup of 217 testees with a follow-up interval of 3 years or more $(r=0.21, P<.01)$. However, to evaluate the impact of the predictive test result on reproduction it is important to focus on the subgroup that is most adequate for this type of analysis: a follow-up interval of at least 3 years and family planning as a major motive in the pretest period. An analysis of covariance aimed at testing the effect of the predictive test result upon having or not having further pregnancies after the predictive test, hereby using 'age at the time of the predictive test' and 'the number of pregnancies before the predictive test' as covariates, clearly revealed that the predictive test result had a significant effect upon having further pregnancies after the predictive test $(F=8.46$; $P<0.01)$. A second analysis of covariance on the same subgroup aimed at additionally testing the main effect of the sex of the testee and the interaction effect 'predictive test result * sex of the testee' (hereby using again age and number of children before predictive testing as covariates) revealed no significant effect of this additional variable and no significant interaction effect. In this second analysis of covariance only the predictive test result had a significant impact $(F=7.03 ; P<0.01)$ on having or not having subsequent pregnancies and the sex of the testee did not play a significant role.

\section{Discussion}

So far less people than expected, based on surveys, have applied for predictive testing for HD. The motivation to have a predictive test is very complex and moreover it is a mixture of conscious and unconscious motives. Although the most frequently mentioned reason is 'having certainty', it is obvious in this study that 'family planning' is an important reason for more than one third of the testees at reproductive age, either in combination with 'having certainty' or as a single reason. Therefore it is interesting to evaluate in a systematic way whether the predictive test result eventually had an impact on post predictive test reproductive decision making and reproduction.

Notwithstanding the fact that there was only a relatively low number of testees with family planning as a reason to apply for predictive testing in some of the participating genetic centres, the European collaborative study provided an answer to the question whether a predictive test result for HD has an effect on subsequent reproduction by comparing the reproduction of carriers and non-carriers of the Huntington mutation. A unique characteristic of this study is that this evaluation is done in persons at reproductive age who had a predictive test after the identification of the Huntington gene and who were counselled in one of the participating genetic centres with a well defined pretest and posttest counselling approach. The collaborative study clearly revealed a measurable impact of the predictive test result on subsequent reproduction: $14 \%$ of the carriers had one or more subsequent pregnancies vs $28 \%$ of the noncarriers. In the total carrier group prenatal diagnosis was 
Table 7 Post predictive test reproduction of non-carriers of the Huntington mutation who expressed family planning as a motive for applying for predictive testing in the pretest period: data per centre

\begin{tabular}{lccccccc}
\hline Non-carriers & Leuven $(\mathrm{n}=20)$ & Cardiff $(\mathrm{n}=12)$ & Paris $(\mathrm{n}=20)$ & Leiden $(\mathrm{n}=10)$ & Athens $(\mathrm{n}=40)$ & Rome $(\mathrm{n}=8)$ Aberdeen $(\mathrm{n}=4)$ & Total $(\mathrm{n}=114)$ \\
\hline $\begin{array}{l}\text { Number of testees with } \\
\text { pregnancies }\end{array}$ & 11 & 1 & 9 & 7 & 30 & 3 & 3 \\
$\quad$ Number of pregnancies & 14 & 1 & 10 & 11 & 45 & 3 & 4 \\
$\quad$ Number of births & 13 & 1 & 5 & 10 & 33 & 3 & 3 \\
Number of miscarriages & 1 & 0 & 4 & 1 & 12 & 0 & 1 \\
Number of terminations & 0 & 0 & 1 & 0 & 0 & 0 & 0 \\
\hline
\end{tabular}

performed in about two thirds of the pregnancies and one couple had chosen for PGD resulting in the birth of a child before the last follow-up contact. In the subgroup who reported 'family planning' as a motive to apply for predictive testing in the pretest period and with a follow-up interval of at least 3 years - the subgroup that is most adequate for this type of evaluation - the effect was more pronounced: $39 \%$ of the carriers of the Huntington mutation had subsequent pregnancies vs $69 \%$ of the non-carriers. Notwithstanding a desire for children in the pretest period and the presence of family planning as one of the reasons to have a predictive test, the majority of carriers had no subsequent pregnancy within a follow-up interval of 3-7 years. A prenatal test was carried out in slightly less than two thirds of the pregnancies in the group of carriers.

For a correct interpretation of the results it is important to keep in mind that 'family planning' as a motive for predictive testing may have a completely different meaning for different predictive test applicants. A first group of people with a desire for children may not want to transmit the Huntington mutation to the next generation and may elaborate different scenarios should the predictive test reveal that they are carrier: refraining from having children, adoption, having pregnancies with prenatal diagnosis or making use of in vitro fertilisation and preimplantation genetic diagnosis. The latter option is very recent and is only available in a few centres in the world. ${ }^{19}$ The alternatives, artificial insemination with donor sperm or in vitro fertilisation with egg cell donation or adoption, were not at all reported during the post predictive test period of the carriers of the Huntington mutation in the present study. A second group may not want to have children, because they do not want to confront their children with a parent who may become affected during their offspring's childhood or adolescence. This conviction may be induced by negative experiences in their own childhood or adolescence. There may of course be an overlap between the two groups. Moreover the weight given to each of the two aspects (transmission of the mutation and impact of an affected parent on the children's education and life) may differ from one couple to another and also between the two members of the same couple or within the same person from one period to another. A third group may need a predictive test result for planning the exact number of children or for timing the birth of their children, eg postpone having children in case of a favourable predictive test result and having children as soon as possible in the case of an unfavourable predictive test result. The former option may for instance be chosen because they have a subjective feeling of 'more time' and the latter opinion may be chosen because of a subjective feeling of time pressure. Taking into account the time pressure that might be felt by part of the carrier group, it can be hypothesised that the difference in reproduction between carriers and noncarriers could even become larger in case of a longer followup interval.

The results of the collaborative study clearly show that predictive DNA-testing helps many couples to fulfil their desire to have (more) children without any risk of transmitting the mutation. Indeed slightly more than two-thirds of the non-carriers with family planning as a motive for predictive testing in the pretest period and who were followed for at least 3 years had one or more subsequent pregnancies. For the carrier group with a desire for (more) children in the pretest period the situation is definitely more complex. The majority of them did not fulfil their desire to have (more) children and did not have any pregnancy within the studied interval of at least 3 years. However a small minority had one or more pregnancies with prenatal diagnosis leading to the birth of several children without the Huntington mutation (as a realisation of their desire to have children without the Huntington mutation). On the other hand the results also show that a slightly smaller group of carriers, with family planning as a motive to apply for predictive testing and/or the intention to avoid the risk of transmitting the disease seem to have changed their mind and had children without prenatal testing. Intentions and behaviour are not always in the same line as has already often been shown in the context of predictive and prenatal testing. Intentions are indeed considered to be one of the stages of decision making preceding actual behaviour (Weinstein). ${ }^{22}$

The present collaborative study has tried to describe the short- to mid-term impact (7 years or less) of a predictive test result on reproduction. A study on a larger group of testees with a desire for children when they apply for predictive testing and with a longer follow-up interval would give a more complete picture. Moreover it would give a better 
opportunity to analyse the intercultural differences. Due to the very small numbers of the adequate subgroup for analysis in some of the participating centres (notwithstanding a relatively large total study group) this issue could not be adequately addressed in the present study.

To put the findings of the present study in a correct perspective it is important to take into account that only a minority of persons at risk for HD make use of predictive testing and prenatal testing. Although systematic research on reproductive decision making of the group who did not apply for predictive testing is lacking, all professionals confronted with families who are aware of their increased risk for Huntington's disease have experienced in practice that reproductive decision making is and remains a difficult dilemma for most of these couples. It is also clear that the final decision (as is the case for many other important life decisions) is not always the result of well structured or rational decision making. Decision difficulties and ambivalence are also illustrated by the fact that some applicants for predictive testing have become pregnant in the months or weeks preceding the communication of the predictive test result. It is clear that a wide variety of emotional factors, transgenerational loyalties, and so far unknown issues, play a part in reproductive decision making in a situation of increased genetic risk for late onset disease. Moreover it can not be excluded that counsellor characteristics may have an impact on decision making of testees, notwithstanding the fact that genetic counselling is in principle nondirective. However this topic was beyond the scope of the present study. There is no evidence in this European study that couples 'take the risk' after being informed that they have the Huntington mutation, because of an optimistic hope for cure. The latter seems a reason for low uptake for prenatal testing in Canada. ${ }^{10}$ Whatever option is chosen by a couple at increased risk of transmitting the Huntingon mutation, it is of the utmost importance that professionals fully respect this decision and support the couple.

\section{Acknowledgments}

The authors are grateful to the many persons in the participating genetic centers without whom the present study would have been impossible: in Leuven (Belgium): Dr M Decruyenaere, Mrs T Cloostermans, Mrs A Boogaerts, Prof Dr G Matthijs and Prof Dr J-P Fryns; in Cardiff (U.K): $\operatorname{Dr} R$ Harper and Mrs $R$ Glew; in Leiden (The Netherlands): Mrs JA Maat-Kievit, Prof Dr MH Breuning, Dr M Losekoot, Prof Dr B Bakker and Mrs MN Ané; in Paris (France): Prof Dr Josué Feingold, Mrs Marcela Garguilo, Mrs Benedicte Prouvost, Mrs Tecla Capecchi and Dr Khadija Lahlou; in Rome (Italy): Dr M Frontali; in Athens (Greece): Prof C Papageorgiou, Prof M Dalakas, Prof D Vassilopoulos, Dr C Voumvourakis, Dr M Panas and Mrs S Pomoni. Very special gratitude is expressed to Mrs N Opdekamp for secretarial assistance during the study and during the preparation of this study.

\section{References}

1 Huntington's Disease Collaborative Research Group: A novel gene containing a trinucleotide repeat that is expanded and unstable on Huntington's disease chromosomes. Cell 1993; 72: 971-983.

2 Harper P, Lim C, Craufurd D: Ten years of presymptomatic testing for Huntington's disease: the experience of the U.K. Huntington's Disease Prediction Consortium. Journal of Medical Genetics 2000; 37: $567-571$.

3 International Huntington Association and World Federation of Neurology: guidelines for the molecular genetics predictive test in Huntington's disease. Neurology 1994; 44: 1533-1536

4 Craufurd D, Dodge A, Kerzin-Storrar L, Harris R: Uptake of presymptomatic predictive testing for Huntington's disease (letter). Lancet 1989; 2: 603-605.

5 Quaid KA, Morris, M: M. Reluctance to undergo predictive testing: the case of Huntington disease. Am J Med 1993; 45: 41 45 .

6 Tibben A, Niermeijer MF, Roos RC, Vegter-Vandervlis M et al. Understanding the low uptake of presymptomatic DNA-testing for Huntington's disease (letter). Lancet 1992; 340: 1416.

7 Evers-Kiebooms G, Decruyenaere M: Predictive testing for Huntington's disease: a challenge for persons at risk and for professionals. Patient Education and Counseling 1998; 35: 15-26.

8 Yapijakis C, Laccone F, Sörenson S. Predictive and prenatal testing for HD in Greece, Germany, Austria, Switzerland and Denmark; in Evers-Kiebooms G, Zoeteweij M, Harper P (eds): Prenatal testing for late onset neurogenetic diseases (2002).

9 Nance $M$. Predictive and prenatal testing for late onset neurogenetic diseases in North America; in Evers-Kiebooms G, Zoeteweij M, Harper P (eds): Prenatal testing for late onset neurogenetic diseases (2002).

10 Hayden MR: Predictive Testing for Huntington Disease - 15 years later. (Presentation during the International Congress of Human Genetics in Vienna, 15 May, 2001).

11 Decruyenaere M, Evers-Kiebooms G, Boogaerts A, Cassiman JJ, Cloostermans T, et al: Non participation in predictive testing for Huntington's disease: individual decision making, personality and avoidment behavior in the family. Eur J Hum Genet 1997; 5: $351-363$.

12 Van der Steenstraten I, Tibben A, Roos RA, Van de Kamp, JJ, Niermeijer MF: Predictive testing for Huntington disease: nonparticipants compared with participants in the Dutch program. Am J Hum Genet 1994; 55: 618-625.

13 Harper P: Huntington's disease. London: Saunders Company, 1996.

14 Tyler A, Quarrell O, Lazarou LP, Meredith AL, Harper PS: Exclusion testing in pregnancy for Huntington's disease. J Med Genet 1990; 27: $488-495$.

15 Adam S, Wiggins S, Whyte P, Bloch M, Shokeir M, Soltan H, et al: (1993) Five year study of prenatal testing for Huntington's disease: demand, attitudes and psychological assessment. J Med Genet 1993; 30: 549-556.

16 Tolmie JL, Davidson HR, May HM, McIntosh K, Paterson JS, Smith B: The prenatal exclusion test for Huntington's disease: experience in the west of Scotland, 1986-1993. J Med Genet 1995; 32: 97-101.

17 Schulman JD, Black SH, Handyside A, Nance WE: Preimplantation genetic testing for Huntington's disease and certain other dominantly inherited disorders. Clin Genet 1996; 49: 57-58.

18 Evers-Kiebooms G, Fryns JP, Demyttenaere K, Decruyenaere M, Boogaerts A et al: Predictive and preimplantation genetic testing for Huntington's disease and other late-onset dominant disorders: not in conflict but complementary (letter) Clin Genet 1996; 50: $275-276$. 
19 Geraedts J, Liebaers I: Preimplantation genetic diagnosis for Huntington's disease; in Evers-Kiebooms G, Zoeteweij M, Harper P (eds): Prenatal testing for late onset neurogenetic diseases. (2002).

20 Evers-Kiebooms G, Welkenhuysen M, Claes E, Decruyenaere M, Denayer L: The psychological complexity of predictive testing for late onset neurogenetic diseases and hereditary cancers. Soc Sci Med 2000; 51: 831-841.
21 SAS Institute Inc.: SAS/STAT User's Guide, Version 8, Cary, NC: SAS Institute Inc., 1999, 3884 pp.

22 Weinstein ND, Rothman AJ, Sutton SR: Stage theories of health behavior: Conceptual and methodological issues. Health Psychol 1998; 17: 290-299. 